\title{
Fission studies by prompt gamma-ray spectrometry
}

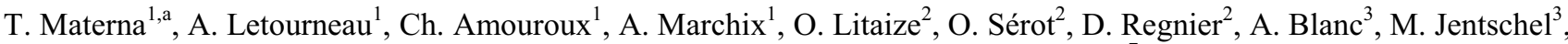 \\ U. Köster ${ }^{3}$, P. Mutti ${ }^{3}$, T. Soldner ${ }^{3}$, G. Simpson ${ }^{4}$, S. Leoni ${ }^{5}$, G. de France ${ }^{6}$ and W. Urban ${ }^{7}$ \\ ${ }^{1}$ IRFU, CEA Saclay, 91191 Gif-sur-Yvette, France \\ ${ }^{2}$ CEA, DEN, Cadarache, 13108 Saint-Paul-lez-Durance, France \\ ${ }^{3}$ ILL, 71 av. des Martyrs, 38042 Grenoble CEDEX 9, France \\ ${ }^{4}$ LPSC, 53 rue des Martyrs, 38026 Grenoble CEDEX, France \\ ${ }^{5}$ INFN Sezione di Milano, I-20133 Milano, Italy \\ ${ }^{6}$ GANIL, BP 55027, 14076 Caen CEDEX 5, France \\ ${ }^{7}$ Faculty of Physics, University of Warsaw, ulica Hoża 69, 00-681 Warsaw, Poland
}

\begin{abstract}
The feasibility of retrieving accurate fission observables with a Ge-detector array around a fissile target placed in a cold neutron beam was tested. In three measurement campaigns performed at ILL with the EXILL setup, ${ }^{235} \mathrm{U}$ and ${ }^{241} \mathrm{Pu}$ targets were placed in the high flux cold neutron beam available at the PF1B neutron guide. Gamma-rays following fission were detected by an array of 16 Ge detectors. In the following study, part of data was analyzed as a proof of principle. A set of yields belonging to the $\mathrm{Kr}$-Ba pair were extracted using a gamma-gamma coincidence technique. Preliminary results were compared to the predictions of two phenomenological models: GEF and FIFRELIN.
\end{abstract}

\section{Introduction}

The complexity of the nuclear fission process makes it difficult to be described precisely by microscopic models. Following the capture of a thermal neutron, the compound nucleus fissions into two different fragments. Most of the energy of the process is transferred into kinetic energy, the fragments being repulsed by Coulomb force according to their shape and average distance at scission. The remaining energy is shared between the two nascent fragments. The majority of this excitation energy is then lost within some picoseconds by the evaporation of a few neutrons and the emission of $\gamma$-rays in cascade. There are several open questions on the fission process that are not yet answered in detail: how exactly the total excitation energy is shared between the fragments; what is the fragment spin distribution and can we infer it from a specific measured cascade; can one describe the prompt decay cascade in a quantitative way.

Practically, to overcome the low accuracy of microscopic models in the prediction of the fission observables, nuclear technology, from reactor operation to nuclear waste, relies on libraries of evaluated data, semiempirical models and "integral" measurements on prototypes. Such a strategy requires a huge number of systematic and accurate experimental data on the few possible observables, among them, yields and kinetic energy (KE) of the fission fragments, neutron and $\gamma$-rays energy and multiplicity, which, sometimes, do not match with integral measurements. A better understanding of the fission process together with better-focused observables is requested to improve the models and the simulation codes.

A unique instrument for the precise study of the fission reaction is the recoil fission fragment mass separator Lohengrin installed at the Institut Laue-Langevin (Grenoble, France). For several decades, it made possible to measure yields and $\mathrm{KE}$ of the light fragments for most actinides in the thermal energy range, with a mass resolution better than 0.3 mass units. In the recent past, this activity was extended to the measurement of the isotopic yields of heavy fragments produced in the ${ }^{235} \mathrm{U}\left(\mathrm{n}_{\mathrm{th}}, \mathrm{f}\right), \quad{ }^{239} \mathrm{Pu}\left(\mathrm{n}_{\mathrm{th}}, \mathrm{f}\right), \quad{ }^{241} \mathrm{Pu}\left(\mathrm{n}_{\mathrm{th}}, \mathrm{f}\right), \quad{ }^{233} \mathrm{U}\left(\mathrm{n}_{\mathrm{th}}, \mathrm{f}\right) \quad$ or ${ }^{242} \mathrm{Am}\left(\mathrm{n}_{\mathrm{th}}, \mathrm{f}\right)$ reactions [1] thanks to a new technique based on delayed $\gamma$-ray spectrometry i.e. after the beta-decay of the fission fragments. However, Lohengrin is limited to the observation of one of the fission fragments only, and the 23-m flight-path of the spectrometer makes, for instance, the study of the prompt decay cascade impossible.

Three EXILL campaigns in 2012-2013 combined a large set of germanium detectors and anti-Compton shields around a thick fissile target, two with ${ }^{235} \mathrm{U}$ and the last one with ${ }^{241} \mathrm{Pu}$. The huge number of double and triple events of prompt $\gamma$-rays in coincidence opens new possibilities in the study of the fission process with new

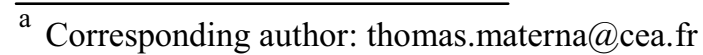


observables of interest also for nuclear technology. Here we report on a study to assess the possibility of retrieving fission fragment pair yields from the data collected with EXILL. Another observable under interest is the $\gamma$-ray cascade itself, i.e. the population of the nuclear level scheme at high energy and angular momentum as a result of the fission process and its decay to the ground state.

\section{State of the art}

The (post-evaporation) fragment-pair distribution is directly related to the prompt neutron evaporation process and thus to the excitation energy of the fragments at scission. Concerning the practical determination of these specific fission yields with the data collected with an array of Ge detectors, several studies exist, at least two independent ones collected with a spontaneous fission source of ${ }^{252} \mathrm{Cf}[2,3]$, one on the fission of ${ }^{232} \mathrm{Th}$ (through the reaction $\left.{ }^{208} \mathrm{~Pb}\left({ }^{18} \mathrm{O}, \mathrm{f}\right)\right)$ [4] and one with low statistics on ${ }^{235} \mathrm{U}\left(\mathrm{n}_{\mathrm{th}}, \mathrm{f}\right)$ [5]. In short, the yield of a couple of fragments - Y(f1,f2) - may be obtained by counting all the coincidences between the transitions to the ground state in $\mathrm{fl}$ with the transitions to the ground state in $\mathrm{f} 2$. Obviously, the most critical point, in term of accuracy, will be i) to find out all the transitions that reach the ground state, ii) to properly correct for coincidence efficiency in the gating process, especially when a triple $\gamma$ coincidence is applied for cleaning purposes. The situation of odd nuclei with a large number of low energy transitions is complex and requires a large amount of data. For instance, the analysis of ${ }^{232} \mathrm{Th}$ [4] was limited to the yields of even-even nuclei.

Considering the large amount of data obtained in the EXILL campaigns, one should be able to determine the post neutron evaporation fragment-pair yields $\mathrm{Y}\left(\mathrm{Z}_{1}, \mathrm{~A}_{1}, \mathrm{Z}_{2}, \mathrm{~A}_{2}\right)$ for a large set of charge splits. These yields can be directly used to validate the best model and tune its free parameters. For instance, figure 1 shows the fragment pair yields calculated with the last version of the GEF code in the case of the $\mathrm{Kr}-\mathrm{Ba}$ split.

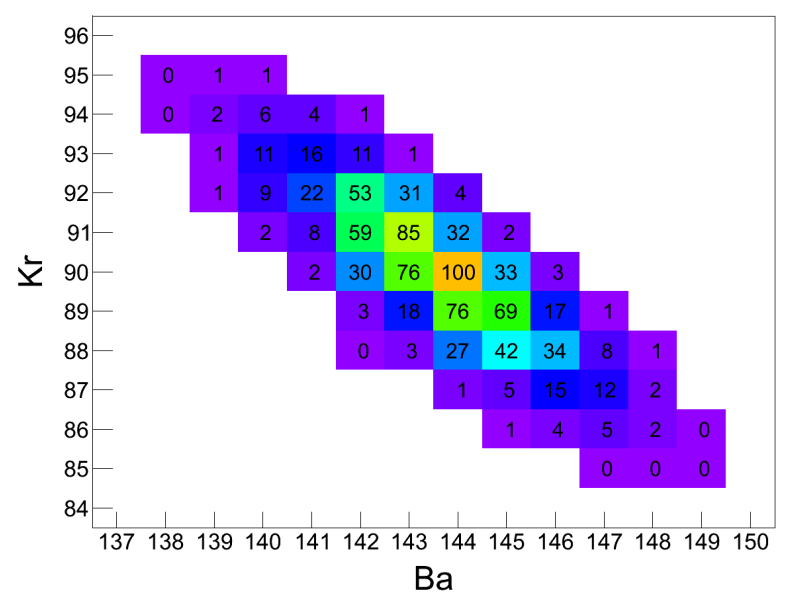

Figure 1. Fission yields of the $\mathrm{Kr}-\mathrm{Ba}$ couples after neutron evaporation according to GEF calculation on the ${ }^{235} \mathrm{U}\left(\mathrm{n}_{\mathrm{th}}, \mathrm{f}\right)$ process. Yields are normalized to the $\left({ }^{90} \mathrm{Kr},{ }^{144} \mathrm{Ba}\right)$ value.

The GEF model [5] is based on a general description of the fission process from a rather general multimodal fission framework and a large empirical knowledge on nuclear data. The GEF code reproduces very well many observables for most studied fissile nuclei in thermal and fast neutron fission like mass yields and kinetic energy of the fragments.

A somewhat similar figure, slightly shifted is obtained using the FIFRELIN code [7]. A major improvement of FIFRELIN versus GEF is the better treatment of the neutron evaporation and $\gamma$-ray cascade. For instance, in contrary to GEF, where only statistical $\gamma$-rays derived from a Fermi based level density were taken into account, FIFRELIN uses (in addition) experimental level schemes from the RIPL-3 database. As a consequence, FIFRELIN reproduces very well the total prompt $\gamma$-ray spectrum emitted at fission [8]. Using EXILL data should allow testing how well performs FIFRELIN on individual $\gamma$-ray cascades and in fine validate the models implemented in this code.

\section{Feasibility study}

Two campaigns were performed on the fission of ${ }^{235} \mathrm{U}$ with cold neutrons from the PF1B beamguide of the ILL, the first one used a $575 \mu \mathrm{g}$ target and lasted 15 days, the second one a $675 \mu \mathrm{g}$ target for about 5 days. The average neutron flux of $10^{8} \mathrm{n} / \mathrm{cm}^{2} / \mathrm{s}$ (after collimation) yields around $10^{5}$ fissions/s. Sixteen germanium detectors were placed around the target at a distance of about $14 \mathrm{~cm}$ : eight EXOGAM detectors, two smaller clovers detectors from ILL and six GASP detectors. The EXOGAM and the GASP detectors were surrounded by BGO active shields. Since the fragments were directly stopped in the target assembly, low Doppler broadening is expected limited to $\gamma$-rays from states with lifetimes below picoseconds. For the same reason, there was no fission fragment detector and as a consequence, no fission trigger helps discriminating between prompt $\gamma$-rays emitted at fission, delayed $\gamma$-rays emitted after $\beta$-decay of the fission fragments and prompt and delayed $\gamma$-rays produced in the surrounded equipment. More details on the experiments may be found in [9].

In this feasibility study, we limited the analysis to the last 15 hours of data collected in the first campaign with ${ }^{235} \mathrm{U}$. In addition, only EXOGAM detectors were considered to avoid corrections in efficiency in the analysis of $\gamma$-rays in coincidence. Detectors were calibrated in energy and efficiency using the data collected with a ${ }^{152} \mathrm{Eu}$ source placed at the target position at the end of the first campaign. Considering the low statistic of this study (4.1\% of the whole set of data on ${ }^{235} \mathrm{U}$ and $65 \%$ of the total detection efficiency), we limited the analysis to the construction of $\gamma-\gamma$ matrix. On the other hand, the $\mathrm{Ba}-\mathrm{Kr}$ pair selected for this study is one of the pairs with the largest yield $-\mathrm{Y}(\mathrm{Kr})=16 \%$ according to JEFF-3.1.1.

For instance the $\gamma$-rays in coincidence with the first excited transition to the ground state of ${ }^{142} \mathrm{Ba}$ (after background subtraction) are shown in Figure 2. Higher excitation transitions in ${ }^{142} \mathrm{Ba}$ are clearly visible in this zoom between 400 and $800 \mathrm{keV}$ as well as main transitions in the complementary fragments ${ }^{90} \mathrm{Kr}$ and ${ }^{92} \mathrm{Kr}$. Once corrected by the relative efficiency of the detection 
system, the intensity of known transitions to the ground states of ${ }^{90} \mathrm{Kr}$ (at 707.1 and $1363 \mathrm{keV}$ ) and ${ }^{92} \mathrm{Kr}$ (at 769 and $1446 \mathrm{keV}$ ) allows us calculating the ratio of the two fission yields $\mathrm{Y}\left({ }^{92} \mathrm{Kr},{ }^{142} \mathrm{Ba}\right) / \mathrm{Y}\left({ }^{90} \mathrm{Kr},{ }^{142} \mathrm{Ba}\right)$. Other ratios were obtained in the same way and when needed internal conversion was taken into account to correct transition intensities. A renormalization to $\left({ }^{90} \mathrm{Kr},{ }^{144} \mathrm{Ba}\right)$ pair yield was done by gating the $\gamma-\gamma$ matrix on ${ }^{90} \mathrm{Kr}$, indeed:

$$
\frac{Y\left({ }^{92} K r,{ }^{142} B a\right)}{Y\left({ }^{90} K r,{ }^{144} B a\right)}=\frac{Y\left({ }^{92} K r,{ }^{142} B a\right)}{Y\left({ }^{90} K r,{ }^{142} B a\right)} \times \frac{Y\left({ }^{90} K r,{ }^{142} B a\right)}{Y\left({ }^{90} K r,{ }^{144} B a\right)}
$$

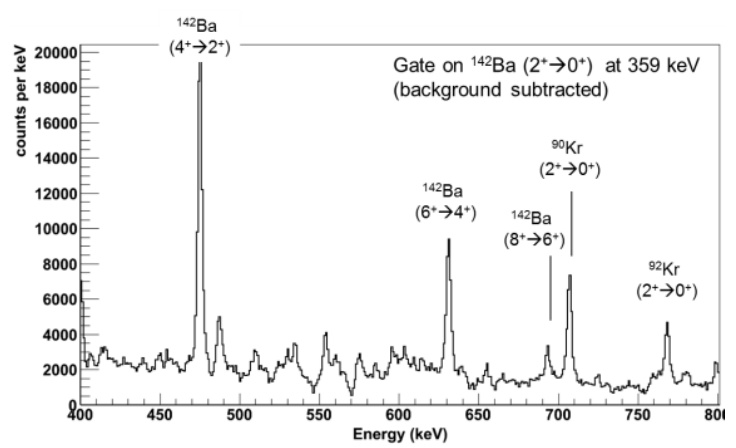

Figure 2. $\gamma$-ray spectrum in coincidence with the $\left(2^{+} \rightarrow 0^{+}\right)$ transition at $359 \mathrm{keV}$ in ${ }^{142} \mathrm{Ba}$. Background subtraction is performed by subtracting the spectrum in coincidence with the energy bin at $363 \mathrm{keV}$.

The analysis finally results in an experimental table of fission pairs associated to the couple $(\mathrm{Kr}, \mathrm{Ba})$ normalised on the $\left({ }^{90} \mathrm{Kr},{ }^{144} \mathrm{Ba}\right)$ yield. It can directly be compared to the tables obtained with the GEF or the FIFRELIN code. Here only a subset of the fragment pair yield table was deduced from the data and is displayed in Table 1. Statistical errors are all lower than 3\%. Systematic errors were not yet well quantified but first estimations based e.g. on the selection of the gates shows they are at least of the order of $20-40 \%$. The same subset was calculated with GEF and FIFRELIN and displayed in Table 2.

Table 1. Experimental yields obtained in this preliminary study normalised to 100 on the couple $\left({ }^{90} \mathrm{Kr},{ }^{144} \mathrm{Ba}\right)$. Values in italics are most probably under-estimated.

\begin{tabular}{|c|c|c|c|}
\hline $\begin{array}{c}\text { Ba } \\
\text { Kr }\end{array}$ & $\mathbf{1 4 2}$ & $\mathbf{1 4 3}$ & $\mathbf{1 4 4}$ \\
\hline $\mathbf{9 2}$ & 48 & 20 & 7 \\
\hline $\mathbf{9 1}$ & 9 & 5 & 5 \\
\hline $\mathbf{9 0}$ & 95 & 81 & 100 \\
\hline $\mathbf{8 9}$ & 5 & 10 & 31 \\
\hline $\mathbf{8 8}$ & 0 & 8 & 17 \\
\hline
\end{tabular}

Experimental yields associated to pairs of even-even nuclei agree surprisingly well with the one obtained with GEF and FIFRELIN. Experimental yields associated to odd-even nuclei are more difficult to obtain and looked often underestimated in our analysis. This effect was related to the selection of possible transitions to the ground state. The case of ${ }^{91} \mathrm{Kr}$ is a good example. For ${ }^{91} \mathrm{Kr}$, we considered the transition at $1155.5 \mathrm{keV}$ only, which was seen in prompt- $\gamma$ decay following fission of
${ }^{252} \mathrm{Cf}[10]$. The $\left(3 / 2^{+} \rightarrow 5 / 2^{+}\right)$transition at $142.3 \mathrm{keV}$ observed in $\beta$-decay and $\beta n$-decay experiments [11] was not clearly identified in our data. As a consequence, it was not included and may explain the underdetermination of the yield. A similar question occurred for ${ }^{89} \mathrm{Kr}$ where we considered the transition at $953.9 \mathrm{keV}$ only. One should add that identifying and quantifying low energy transitions associated to these nuclei seems only possible when the spectra are "clean" at low energy, which is not the case when the analysis is performed only by a simple $\gamma-\gamma$ coincidence. One may expect cleaner spectra from $\gamma-\gamma-\gamma$ coincidence.

Table 2. Yields calculated with respectively GEF (left) and FIFRELIN (right) for the ${ }^{235} \mathrm{U}\left(\mathrm{n}_{\mathrm{th}}, \mathrm{f}\right)$ reaction with the same normalisation as in Table 1.

\begin{tabular}{|c|c|c|c|c|c|c|c|}
\hline $\begin{array}{c}\text { Ba } \\
\text { Kr }\end{array}$ & $\mathbf{1 4 2}$ & $\mathbf{1 4 3}$ & $\mathbf{1 4 4}$ & & $\mathbf{1 4 2}$ & $\mathbf{1 4 3}$ & $\mathbf{1 4 4}$ \\
\hline $\mathbf{9 2}$ & 53 & 31 & 4 & & 78 & 14 & 10 \\
\hline $\mathbf{9 1}$ & 59 & 85 & 32 & & 92 & 111 & 38 \\
\hline $\mathbf{9 0}$ & 30 & 76 & 100 & & 82 & 123 & 100 \\
\hline $\mathbf{8 9}$ & 3 & 18 & 76 & & 28 & 56 & 80 \\
\hline $\mathbf{8 8}$ & 0 & 3 & 27 & & 1 & 15 & 29 \\
\hline
\end{tabular}

\section{Conclusions and Perspectives}

The feasibility study performed on a tiny set of data collected during the EXILL campaign with the ${ }^{235} \mathrm{U}$ target shows that it is possible to retrieve the post-neutron evaporation yields of fragment pairs produced at fission when fragments are even-even nuclei. Difficulties were encountered for odd-even and odd-odd nuclei and were not solved. Experimental yields agreed reasonably well with the two most recent phenomenological models GEF and FIFRELIN. In this study, the analysis was performed only with $\gamma-\gamma$ coincidence due to the low statistics. The complete analysis will be done using triple $\gamma$ coincidence. Cleaner spectra are then expected. However, it may not directly solve the problem of unknown transitions to the ground state. It was not intended with this feasibility study to discriminate between the best implementation of the neutron evaporation. This will be one of the purposes of the complete analysis.

\section{References}

1. O. Serot et al., Nuclear Data Sheet 119, 320 (2014)

2. G.M. Ter-Akopian et al., Phys. Rev. Lett 73, 1477 (1994)

3. D. Fong et al., Phys. At. Nucl. 69, 1161 (2006)

4. A. Bogachev et al., Eur. Phys. J. A 36, 23 (2007)

5. S. Mukhopadhyay et al., Phys. Rev. C 85, 064321 (2012)

6. K-H. Schmidt, B. Jurado and Ch. Amouroux, JEFFReport 24, NEA / DB / DOC (2014)

7. O. Litaize, O. Serot, Phys. Rev. C 82, 054616 (2010)

8. D. Regnier et al., Phys. Proc. 47, 47 (2013)

9. A. Blanc et al., EPJ Conf. 62, 01001 (2013)

10. J. K. Hwang et al, Phys. Rev. C 78, 017303 (2008)

11. C. M. Baglin, Nuclear Data Sheet 114, 1293 (2013) 
